\title{
What did Syndromic Surveillance Show During London 2012? Lessons for Mass Gatherings
}

\author{
Dan Todkill ${ }^{1}, 2$, Helen Hughes*1, Alex Elliot ${ }^{1}$, Roger Morbey ${ }^{1}$, Obaghe Edeghere ${ }^{1}$, Sally \\ Harcourt ${ }^{1}$, Brian McCloskey ${ }^{2}$ and Gillian Smith ${ }^{1}$
}

${ }^{1}$ Public Health England, Birmingham, United Kingdom; 'Public Health England, London, United Kingdom

\section{Objective}

We assessed the impact of the London 2012 Olympic and Paralympic Games on syndromic surveillance systems including the incidence of syndromic indictors and total contacts with health care.

\section{Introduction}

Mass gatherings can impact on the health of the public including importation of infectious diseases, exposure of international visitors to endemic diseases in the host country and the increased risk of bioterrorist activity. ${ }^{1}$ Public health surveillance during mass gatherings therefore affords an opportunity to identify, and quantify any impact (or reassure on the absence of impact) on public health in a timely manner.

In preparation for the Games, Public Health England undertook a programme of work to expand the existing suite of syndromic surveillance systems to include daily general practitioner out of hours $(\mathrm{GPOOH})$ consultations and emergency department (ED) attendances at sentinel sites. ${ }^{2}$ These new systems complemented existing syndromic surveillance systems offering the opportunity to monitor trends in patient contacts with GPs outside of normal day time opening hours, as well as potentially the more severe end of the disease spectrum which would present at EDs.

We assessed the impact of the 2012 Olympics on national surveillance systems, comparing to periods before and after the Games and in previous years and also the impact of specific events during the Games.

\section{Methods}

The daily syndromic surveillance data gathered during the Olympic period 2012 were compared to the comparable surveillance data collected throughout the period preceding and following, and equivalent time period in 2013.

\section{Results}

The principal finding was that very few differences were found between years, within both the ED and GPOOH systems, nationally or within London. Some exceptions were noted; insect bite and sting related indicators were at higher levels during and immediately after the Olympic period in 2012 than the equivalent time period in 2013 and an increase in attendances for acute alcohol consumption related diagnoses were observed in London EDs around the Olympic opening ceremony. A reduction in total daily ED attendances and GPOOH contacts during summer 2012 was observed, but a similar pattern was observed in the following year and this appears to be a secular trend coinciding with school holidays.

\section{Conclusions}

The 2012 London Olympic Games had very little obvious impact on the daily number of ED attendances and GPOOH contacts both nationally and within London, with what has now been observed to be a normal summer reduction observed across both systems. This highlights the importance for any surveillance system to be used for a mass gathering to have a sufficient amount of baseline information from the same time period in at least one previous year to aid in the identification of any departures from what is considered to be 'normal' within each system, at each location at that time of the year.

The ED and GPOOH systems provided valuable reassurance of no emerging public health issues during the Games, with the two items found to have been at higher levels than 2013 being those related to non-infectious causes (i.e. bites and stings) and only one of these having a potential link to the Games itself (acute alcohol related ED attendances around the timing of the opening ceremony).

\section{Keywords}

syndromic surveillance; mass gathering; Olympic Games; baseline

\section{References}

1. Tsouros AD, Efstathiou PA. Mass gatherings and public health - the experience of the Athens 2004 Olympic Games. 2007. http://www. euro.who.int/document/e90712.pdf (accessed 13 February 2013).

2. Elliot AJ, Morbey RA, Hughes HE, et al. Syndromic surveillance - a public health legacy of the London 2012 Olympic and Paralympic Games. Public Health 2013;127:777-81.

\section{*Helen Hughes}

E-mail: helen.hughes@phe.gov.uk 